\title{
Orthodontics in the future
}

\author{
S. Lacroix
}

Editor-in-chief of the Union

Beware, this is purely a work of fiction. Let us imagine that we are in the year 2085. Specifically, the 8th of March at 7:00 a.m. It is still international women's day, nothing has changed in this regard. Sylvie, a mom, has programmed everything the night before. Soft lights gradually light up the house. Jim, the family robot, has set the table and has prepared fresh coffee and croissants. Little Alain, 12 years old, stretches in his bed, lights his planiscope with voice control. A soft female voice runs through his daily schedule. She also reminds him that he has not consulted his e-health application for almost 2 months. Trying to avoid it would be futile. Mom Sylvie comes to kiss him on the forehead and confirms that he will have to undergo a health check tonight. Jim the robot is sterner and thinks that medical follow-up is serious business. Nothing escapes Jim the robot. This is because he constantly receives all the information transmitted to him by the chip found under Alain's skin, between his fifth and sixth ribs, via Wi-Fi. Jim the robot alerts the little boy to even the slightest changes from his health model stored in the cloud. Unstoppable.

At 5:00 p.m., as soon as he has finished supper, Alain sits down with Jim in his room for a one-on-one interview. The robot reviews all the data it has at its disposal. At the same time, figures and graphs appear on the screen of its flexible tablet. All is well, Jim goes quickly from one line of questioning to another. It stops when it reaches "orthodontics." Instead the figures flash red. Alain clicks on the tab, the instructions are clear: "Make an appointment with an orthodontist." Alain clicks again and is directed to the nearest orthodontic center. $\mathrm{He}$ checks the timetable at 3:30 p.m., the next afternoon, and types in his password.

The orthodontics center is a new building with all the latest innovations. The reception area has been simplified significantly. It consists of a simple terminal which automatically recognizes Alain thanks to his chip. He walks into the main hallway and sees on a screen that his consultation will take place on the 3rd floor, in room 21. When he enters the room, decorated in pastel colors, he is alone. It takes a seat in the large armchair and takes a few moments to contemplate the ceiling covered with a huge plasma screen where beautiful pictures of landscapes transition in and out. Meanwhile, six infrared and laser cameras focus in on his face. They are focusing on his maxilla, recording even the most imperceptible of movements. The images are

Address for correspondence:

Sébastien Lacroix - 14, rue Edouard Mignot - 51100

Reims

E-mail: slacroix@journal-lunion.fr

Article Received: 05-09-2016. Accepted for publication: 18-11-2016.

This is an Open Access article distributed under the terms of the Creative Commons Attribution License (http://creativecommons.org/licenses/by/4.0), which permits unrestricted use, distribution, and reproduction in any medium, provided the original work is properly cited. 
transmitted to the centralized system that analyzes them in real time. A few minutes are enough for Alain's dental profile to be updated.

A little beep sounds and a young woman enters the room. You would think she came straight from a space station: Pink technical clothing and an augmented reality mask on her forehead. From her belt, she extracts a kind of pen and she moves the fluorescent end along Alain's teeth, from the maxilla to the mandible. He vaguely listens to her comments, still entranced by the images on the ceiling. The young woman places a tablet next to her. From the curved screen, a hologram showing the upper and lower side of the young boy's mouth gradually materializes.

The next step is to place a vestibular self-adhesive plate on the maxilla and mandible, as well as a pellet on the right and left sides, in front of the ears. Alain makes some lateral propulsion movements, which are faithfully reproduced in 3D before the young woman. The central system indicates that all of the data has been recorded.

The young woman then initiates the $3 \mathrm{D}$ printing of a device, simply by touching the screen. On the left, you can hear the hum of the 3D printer. After a short while, two transparent ceramic arches have been produced. They include all the information necessary to move the teeth. Meticulously, the young woman slips them into Alain's mouth and places them without further intervention as they are precoated with a quick fixation gel.

To finalize the procedure, the young woman places two horseshoe plates in Alain's mouth. They emit rays that will soften the bone. All we have to do is wait. Half an hour later, the teeth are in place, perfectly matching the diagram shown on the central system. The plates are removed, the bone returns to its original texture.

All procedures have been recorded. The young woman reviews them one last time, validating each step simply by touching the screen. The results are excellent. Alain looks in the mirror. "With a smile like that, you will wreak havoc in college," says the young woman. She is already heading to room 22 where the patient is waiting for a $4: 15$ p.m. appointment. And when it comes to paying fees. Alain enters his password again in the output terminal. It confirms everything and he leaves the center. Currency has not been in use for a long time so the central system is free.

Conflict of interest: The author states there is no conflict of interest. 